This item was submitted to Loughborough's Research Repository by the author.

Items in Figshare are protected by copyright, with all rights reserved, unless otherwise indicated.

\title{
Chattering-free sliding mode control with unidirectional auxiliary surfaces for miniature helicopters
}

\section{PLEASE CITE THE PUBLISHED VERSION}

http://dx.doi.org/10.1108/17563781211255925

PUBLISHER

(C) Emerald

VERSION

AM (Accepted Manuscript)

\section{LICENCE}

CC BY-NC-ND 4.0

\section{REPOSITORY RECORD}

Fu, Jian, Wen-Hua Chen, and Qing-Xian Wu. 2012. "Chattering-free Sliding Mode Control with Unidirectional Auxiliary Surfaces for Miniature Helicopters”. figshare. https://hdl.handle.net/2134/11108. 
This item was submitted to Loughborough's Institutional Repository (https://dspace.lboro.ac.uk/) by the author and is made available under the following Creative Commons Licence conditions.

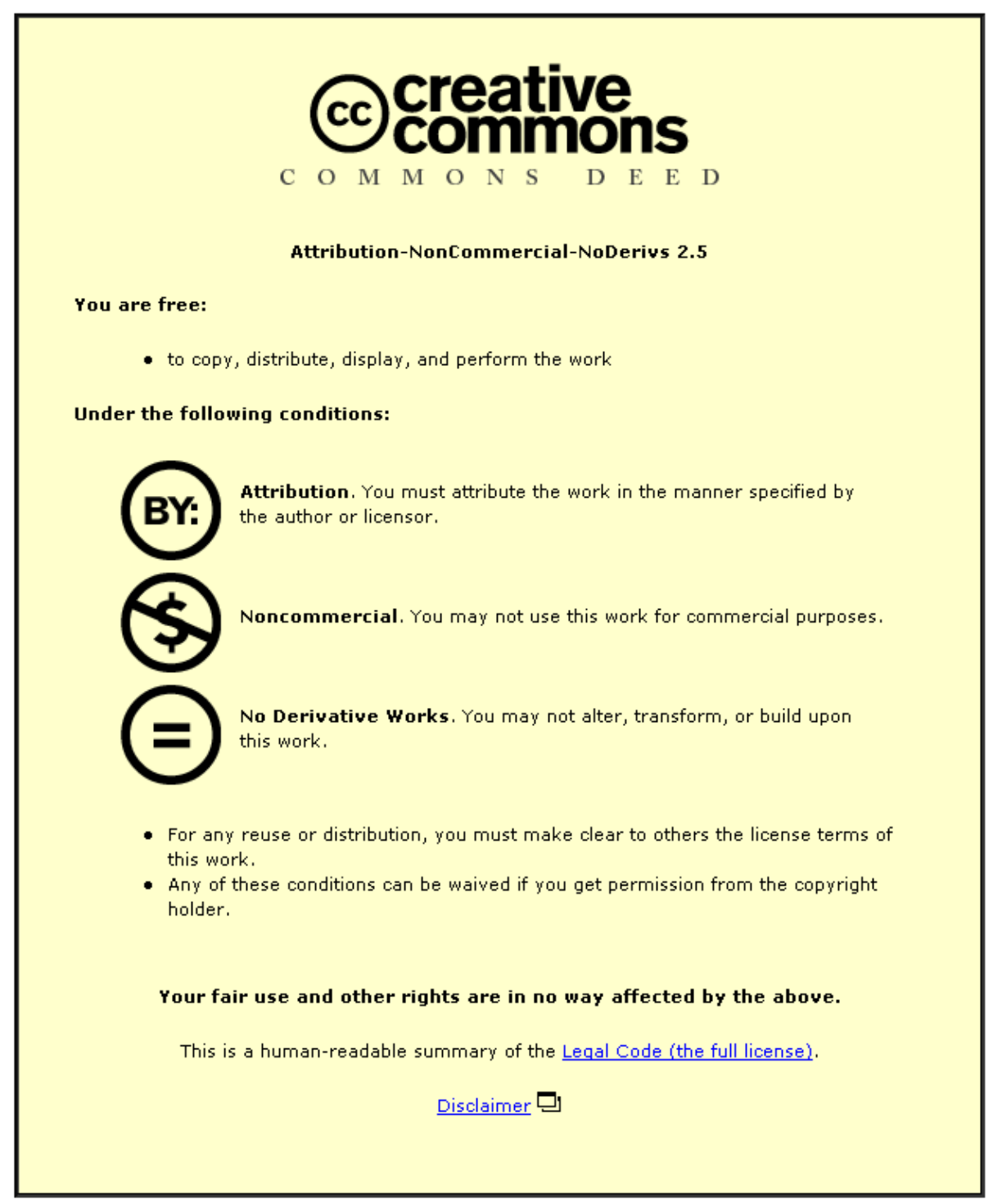

For the full text of this licence, please go to: http://creativecommons.org/licenses/by-nc-nd/2.5/ 


\title{
CHATTERING-FREE SLIDING MODE CONTROL WITH UNIDIRECTIONAL AUXILIARY SURFACES FOR MINIATURE HELICOPTERS
}

\author{
Jian $\mathrm{Fu}^{*}$ \\ Department of Automation, Nanjing University of Aeronautics and Astronautics, \\ Nanjing, China \\ fujian1986216@126.com ${ }^{\ddagger}$ \\ Wen-hua Chen \\ Department of Aeronautical and Automotive Engineering, Loughborough University , \\ Loughborough, UK \\ w.Chen@lboro.ac.uk \\ Qing-xian $\mathrm{Wu}$ \\ Department of Automation, Nanjing University of Aeronautics and Astronautics, \\ Nanjing, China \\ wuqingxian@nuaa.edu.cn
}

Received (Day Month Year)

Revised (Day Month Year)

Accepted (Day Month Year)

\section{Abstract}

This article proposes a chattering-free sliding mode control scheme with unidirectional auxiliary surfaces (UAS-SMC) for small miniature autonomous helicopters (Trex 250). The proposed UAS-SMC scheme consists of a nested sequence of rotor dynamics, angular rate, Euler angle, velocity and position loops. It is demonstrated that the UAS-SMC strategy can eliminated the chattering phenomenon exhibiting in the convenient SMC method and achieve a better approaching quality. The proposed control strategy is implemented on the helicopter and flight tests clearly demonstrate that a much better performance could be achieved, compared with convenient SMC schemes.

Keywords Unmanned aircraft; approaching quality; flight control; sliding mode control; helicopter;

chattering

Paper type

Research paper

\section{Introduction}

Sling mode control (SMC) has long been recognized as a robust method against uncertainty and external disturbances. Many elaborate works in this area have been presented in the literature (Gao, 1996; Bartibkubu et al., 1995; Wei et al., 2008; Michael et al., 2008). In general, a conventional sliding mode control scheme is designed in such a way that stat trajectories are forced to approaching pre-specified sliding surfaces. A 
discontinuous control is designed to achieve approaching quality in the conventional SMC. However, the drawback of this strategy is the chattering problem which may significantly degrades the performance and may even lead to instability.

To preserving the main advantages of the conventional SMC, two most widely known methods, namely higher order SMC and SMC with boundary layers have been proposed to alleviate the chattering phenomenon. The proposed strategies successfully eliminate the chattering phenomenon but they also bring weakness with them. It is quite difficult to use the higher order SMC when a significant level of noise is present since the derivative terms may be required from the measurements (Chen et al., 2002; Lei et al., 2010; Allamehzadeh and Cheung, 2003). The SMC with boundary layers is essentially a kind of quasi-sliding mode control methods (Francesco and Antonella, 2009; A. Gaaloul and F. M' Sahli, 2009) where the discontinuous control activity is replaced by a continuous control effort inside a pre-defined boundary layer around the switching surfaces. Consequently, its robust performance would inevitably be deteriorated with this augmented boundary-layer (Yang et al., 2008).

In general, the higher order SMC and SMC with boundary layers are focused on the chattering problem on the switching surfaces. This paper aims to show that UAS-SMC strategy (Fu et al., 2011b) could eliminate the chattering without pay the price on the approaching quality. The proposed control scheme is designed to tailor the physical structure of the helicopter dynamics. The autonomous flight control problem for a helicopter is divided in several channels, each of which enjoys a cascade structure from the inner loops to the outer loops. The proposed UAS-SMC has been used to design the controller for each subsystem in a sequential manner. This results a cascade UAS-SMC control structure. The proposed control scheme is tested in a dedicated, real flight test environment, together with a conventional SMC scheme. The work presented here is an extension of the work in (Fu et al., 2011a) with an emphasis on chattering behaviour and approaching quality. The rest of this paper is designed as follow: Section 2 contains the analysis of the UAS-SMC and conventional SMC strategies. Section 3 explains the design of the UAS-SMC for helicopters and the experimental environment and setting, whiles Section 4 givens experimental results and the analysis. Finally, this paper is closed with conclusions in Section 5.

\section{UAS-SMC and Conventional Sliding Mode}

\subsection{Conventional sliding mode}

Consider the system in Equation (1)

$$
\dot{X}=f(X)+g(X) u .
$$

where $f(X) \in R^{n}, g(X) \in R^{n \times n}$ is an invertible matrix, the elements in $f(X)$ and $g(X)$ are continuous, $X=\left[x_{1}, \cdots, x_{n}\right]^{T} \in R^{n}$ is the system states and $u \in R^{n}$ is control input. For the sake of clarity, Equation (1) is chosen to show the difference between the conventional SMC and UAS-SMC. Actually, UAS-SMC is applicable to systems with the number of control inputs less than that of states. But this will significantly increase the complexity in our discussion (Fu et al., 2011c).

Figure 1 here 
The formula of stable switching surface $S_{i}, i=1, \cdots, n$ shown in Figure 1 is given as follow:

$$
S_{i}=x_{i}+\omega_{i} \int x_{i} d t
$$

State trajectory $x_{i}$ is forced to approaching the switching surface $S_{i}$ by the approaching law in Equation.(3).

$$
\dot{S}_{i}=-K_{i} \cdot \operatorname{sign}\left(S_{i}\right)
$$

where $K_{i}>0$.

Based on the approaching law in Equation.(3), the conventional sliding mode control for system (1) can be expressed as follow:

$$
u=g^{-1}(X)(-f(X)-\omega \cdot X-K \cdot \operatorname{sign}(S))
$$

where

$$
\omega=\operatorname{diag}\left\{\omega_{1}, \cdots, \omega_{n}\right\}, K=\operatorname{diag}\left\{K_{1}, \cdots, K_{n}\right\}, \operatorname{sign}(S)=\left[\operatorname{sign}\left(S_{1}\right), \cdots, \operatorname{sign}\left(S_{n}\right)\right]^{T}
$$

Since the approaching law $K \cdot \operatorname{sign}(S)$ in Equation (4) is a discontinuous function, the control input $u$ may become discontinuous. To avoid this situation, a smooth function $K \cdot \operatorname{sat}(S)$ is used to replace the switching function $K \cdot \operatorname{sign}(S)$ where $\operatorname{sat}(S)=\left[\operatorname{sat}\left(S_{1}\right), \cdots, \operatorname{sat}\left(S_{n}\right)\right]^{T} . \operatorname{sat}\left(S_{i}\right)$ is the saturation function of $S_{i}$ as given in Equation(5).

$$
\operatorname{sat}\left(S_{i}\right)=\left\{\begin{array}{cc}
1 & S_{i}>1 \\
-1 & S_{i}<-1 \\
S_{i} & \text { others }
\end{array}\right.
$$

In this paper, the approaching quality means the approaching speed and the ability to against the disturbance in the approaching process. Hence, the approaching law $K_{i} \cdot \operatorname{sat}\left(S_{i}\right)$ is used to express the approaching quality for conventional sliding mode strategy. If function $K_{i} \cdot \operatorname{sat}\left(S_{i}\right)=0$, it implies that the approaching quality is low. To avoid confusion, the sign of approaching law is removed. Thus, we obtained function $N_{i}=\left|K_{i} \cdot \operatorname{sat}\left(S_{i}\right)\right|$ to show the approaching quality with chattering-free conventional sliding mode control. The symbol $|\cdot|$ means the absolute value. Higher value in $N_{i}$ implies that the control can against more disturbance in the approaching process. The value of function $N_{i}$ for the switching surface $S_{i}$ in conventional sliding mode control is shown as follow.

\section{Figure 2 here}

The value of function $N_{i}$ in state space $\left(x_{i}, \int x_{i}\right)$ is shown in Figure 2. It is noted that the function $N_{i}=0$ when state $x_{i}$ is sliding on the switching surface $S_{i}$ (The grayscale is used to show the value of function $N_{i}$ for each point in state space. A white point means its corresponding value is zero). Hence, the approaching quality on the switching surface is low. This is the main problem in the conventional sliding mode strategy. The 
approaching quality is high when a switching function is used in the control law. But this introduces the chattering phenomenon. In contrast, when a smooth function is used to replace the switching function, the approaching quality is low (J,J. Slotine et al.,1983; Yigeng et al.,2010).

\subsection{UAS-SMC}

\section{Figure 3 here}

The schematic of UAS-SMC strategy is shown in Figure 3, where the state trajectories are forced to approaching the stable switching surfaces $S_{1 i}, S_{2 i}$ with unidirectional auxiliary surfaces defined by $H_{0 i}, H_{1 i}, H_{2 i}, H_{3 i}=0$ where

$$
\begin{gathered}
S_{1 i}=x_{i}+\xi_{1 i} \int x_{i} ; S_{2 i}=x_{i}+\xi_{2 i} \int x_{i} ; \xi_{1 i} \neq \xi_{2 i} \\
H_{k i}=\omega_{k i 1} x_{i}+\omega_{k i 2} \int x_{i}+M_{k i}, M_{k i}>0, k=0,1,2,3
\end{gathered}
$$

The detailed design and analysis process of $S_{1 i}, S_{2 i}, H_{0 i}, H_{1 i}, H_{2 i} H_{3 i}$ can be found in Fu et al. (2011b).

To achieve the UAS-SMC controller, the currently unidirectional auxiliary surface $H_{i}$ is defined as follow:

$$
H_{i}=\omega_{i 1} x_{i}+\omega_{i 2} \int x_{i}+M_{i}
$$

where

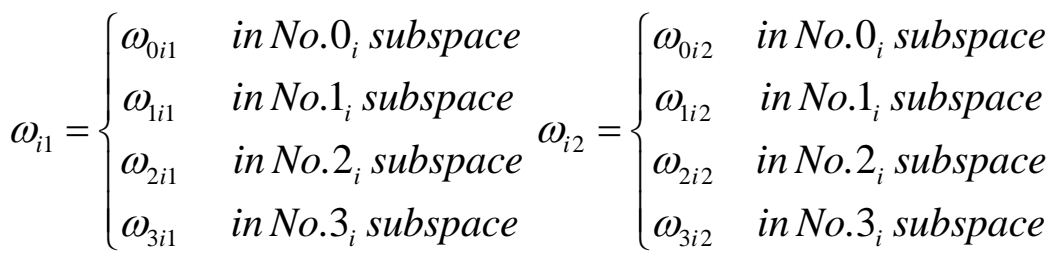

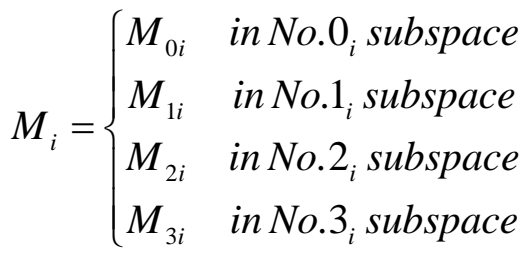

Then, the state trajectory $x_{i}$ is forced to approaching the switching surfaces $S_{1 i}, S_{2 i}$ by Equation (8)

$$
\dot{H}_{i}=\omega_{i 1} \dot{X}_{i}+\omega_{i 2} X_{i}=N_{i}
$$

where $N_{i} \geq 0$ is the approaching law for switching surfaces $S_{1 i}, S_{2 i}$. Since the $N_{i}$ is a positive value, it is directly used to show the approaching quality in UAS-SMC strategy. Define $N=\left[N_{1}, \cdots, N_{i}, \cdots, N_{n}\right]^{T}$, the UAS-SMC control for system (1) can be expressed as follow: 


$$
u=g^{-1}(X)\left(-f(X)+\Omega_{1}^{-1} \cdot N-\Omega_{1}^{-1} \cdot \Omega_{2} \cdot X\right)
$$

where $\Omega_{1}=\operatorname{diag}\left\{\omega_{11}, \cdots, \omega_{i 1}, \cdots, \omega_{n 1}\right\}, \Omega_{2}=\operatorname{diag}\left\{\omega_{12}, \cdots, \omega_{i 2}, \cdots, \omega_{n 2}\right\}$

Since the elements in $f(X)$ and $g(X)$ are continuous, the control $u$ would become continuous function if the continuity of $\Omega_{1}^{-1} \cdot N-\Omega_{1}^{-1} \cdot \Omega_{2} \cdot X$ is guaranteed. From previous discussion, it is noted that

$$
\Omega_{1}^{-1} \cdot N-\Omega_{1}^{-1} \cdot \Omega_{2} \cdot X=\left[\omega_{11}^{-1} N_{1}-\omega_{11}^{-1} \omega_{12} x_{1}, \cdots, \omega_{n 1}^{-1} N_{n}-\omega_{n 1}^{-1} \omega_{n 2} x_{n}\right]^{T}
$$

Take the element $\omega_{i 1}^{-1} N_{i}-\omega_{i 1}^{-1} \omega_{i 2} X_{i}, i=1, \cdots, n$ in Equation (10) as an example, the discussion is given as follow:

When state $X_{i}$ is moving in No. $0_{\mathrm{i}}$ subspace, from Equation (7) it is noted that

$$
\omega_{i 1}^{-1} \cdot N_{i}-\omega_{i 1}^{-1} \cdot \omega_{i 2} \cdot x_{i}=\omega_{0 i 1}^{-1} \cdot N_{0 i}\left(x_{i}\right)-\omega_{0 i 1}^{-1} \cdot \omega_{0 i 2} \cdot x_{i}
$$

where $N_{0 i}\left(x_{i}\right)$ means the continuous function of approaching law $N_{i}$ in No. $0_{\text {i }}$ subspace. When state $x_{i}$ is sliding on switching surface $S_{2 i}$, there exists $N_{0 i}\left(x_{i}\right)=N_{0 i+}$ as shown in Figure 4. Meanwhile, when state $x_{i}$ is moving on switching surface $S_{1 i}$, there exists $N_{0 i}\left(X_{i}\right)=N_{0 i-}$. As the approaching law $N_{i}$ is a function, not a constant value, different parameters can be selected in different subspaces as long as they have positive values.

When state $X_{i}$ is moving in No. $1_{i}$ subspace, from Equation (7) it is noted that

$$
\omega_{i 1}^{-1} \cdot N_{i}-\omega_{i 1}^{-1} \cdot \omega_{i 2} \cdot x_{i}=\omega_{1 i 1}^{-1} \cdot N_{1 i}\left(x_{i}\right)-\omega_{1 i 1}^{-1} \cdot \omega_{1 i 2} \cdot x_{i}
$$

where $N_{1 i}\left(x_{i}\right)$ means the continuous function of approaching law $N_{i}$ in No. $1_{\mathrm{i}}$ subspace. When state $x_{i}$ is sliding on switching surface $S_{2 i}$, there exists $N_{1 i}\left(x_{i}\right)=N_{1 i-}$ as shown in Figure 4. Meanwhile, when state $x_{i}$ is moving on switching surface $S_{1 i}$, there exists $N_{1 i}\left(x_{i}\right)=N_{1 i+}$.

From Equation (11) and Equation (12), there may exist $N_{0 i+} \geq 0, N_{1 i_{-}} \geq 0$ satisfied Equation (13) when state $X_{i}$ is sliding on the switching surface $S_{2 i}$ between No. $0_{\mathrm{i}}$ subspace and No. $1_{\mathrm{i}}$ subspace.

$$
\omega_{0 i 1}^{-1} \cdot N_{0 i+}-\omega_{0 i 1}^{-1} \cdot \omega_{0 i 2} \cdot x_{i}=\omega_{1 i 1}^{-1} \cdot N_{1 i-}-\omega_{1 i 1}^{-1} \cdot \omega_{1 i 2} \cdot x_{i}
$$

Similar situations can be found in No. $2_{\mathrm{i}}$ and No. $3_{\mathrm{i}}$ subspace. For the sake of simplicity, the sufficient conditions for the existence of $N_{0 i+}$ and $N_{1 i-}$ satisfying Equation (13) will not be discussed in this paper.

\section{Figure 4 here}

Analogously, we obtain the approaching law $N_{0 i+}, N_{0 i-}, N_{1 i+}, N_{1 i-}, N_{2 i+}, N_{2 i-}$, $N_{3 i+}, N_{3 i-}$ in Figure 4 which guarantee the continuity of element $\omega_{i 1}^{-1} N_{i}-\omega_{i 1}^{-1} \omega_{i 2} X_{i}$ $, i=1, \cdots, n$ in Equation (10) on the switching surfaces $S_{1 i}, S_{2 i}$ as shown in Figure 4. It is noted that the approaching law $N_{i}$ could be designed as follow: 


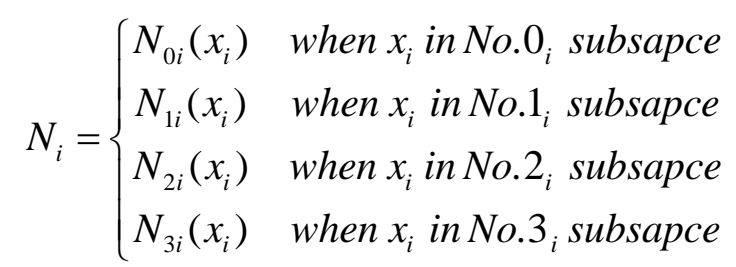

where functions $N_{0 i}\left(x_{i}\right), N_{1 i}\left(x_{i}\right), N_{2 \mathrm{i}}\left(x_{i}\right), N_{3 i}\left(x_{i}\right)$ are continuous in the subspaces.

From Equation (7), it is noted that the coefficients $\omega_{i 1}, \omega_{i 2}$ are constant values when state $X_{i}$ is moving in the subspaces. This, together with the fact that the robust function $N_{i}$ is a continuous function in the subspaces, implies the terms $\omega_{i 1}^{-1} N_{i}-\omega_{i 1}^{-1} \omega_{i 2} x_{i}, i=1, \cdots, n$ in Equation (10) are also continuous in the subspaces.

Based on the previous discussion, the elements $\omega_{i 1}^{-1} N_{i}-\omega_{i 1}^{-1} \omega_{i 2} X_{i}, i=1, \cdots, n$ in Equation (10) are continuous both in the subspaces and on the switching surfaces $S_{1 i}, S_{2 i}$ . Thus, the elements in vector $\Omega_{1}^{-1} \cdot N-\Omega_{1}^{-1} \cdot \Omega_{2} \cdot X$ are continuous. Invoking this fact into Equation (9) and taking into account that the elements in $f(X)$ and $g(X)$ are continuous, one concludes the continuity of control $u$. The chattering phenomenon is caused by the discontinuous control input. Hence, the control $u$ in Equation (9) can be considered as a chattering-free UAS-SMC with approaching law

$$
N=\left[N_{1}, \cdots, N_{i}, \cdots, N_{n}\right]^{T}
$$

The value of approaching law $N_{i}$ for the switching surface $S_{1 i}, S_{2 i}$ in chattering-free UAS-SMC is shown as follow.

\section{Figure 5 here}

Comparing Figure 2 and Figure 5, it is noted that the value of robust function $N_{i}$ is zero in Figure 5 when state $x_{i}$ stays on the original point (The grayscale is used to show the value of function $N_{i}$ for each point in state space. The while point means the value is zero). When the state trajectory is sliding on the switching surfaces $S_{1 i}, S_{2 i}$ with chattering-free UAS-SMC control, the value of approaching law is not zero (The points are not white, except the original point). Hence, the chattering problem around the switching surfaces is transformed into the problem around the original point.

\section{SMC for helicopters}

\subsection{Trex-250 helicopter}

The Trex-250 helicopter used in this paper is shown in Figure 6. It is a small sized helicopter with the main rotor diameter of $460 \mathrm{~mm}$ and the trail rotor diameter of $108 \mathrm{~mm}$. The belt-driven tail and collective pitch rotor make is capable of 3D maneuvers such as inverted flight. It means that it is well-suited for indoor flight test.

\section{Figure 6 here}

The nested controller is developed with mathematical model obtained from Liu et al. (2010a). For the application to Trex-250, we use differential equations as given in Equation (15)-(20). It is obviously that these equations can fall into five different loops, which are position loop (15); velocity loop (16); Euler angle loop (17); angular rate loop 
(18); and rotor dynamic loop (19). According to these loops, a nested controller is proposed. The detail information of math model is expressed as follow.

$$
\begin{gathered}
\dot{x}=(\cos \theta \cdot \cos \psi) \cdot u+(\sin \varphi \cdot \sin \theta \cdot \cos \psi-\cos \varphi \sin \psi) v+ \\
(\cos \varphi \sin \theta \cos \psi+\sin \varphi \sin \psi) w \\
\dot{y}=(\cos \theta \cdot \sin \psi) \cdot u+(\sin \varphi \cdot \sin \theta \cdot \sin \psi+\cos \varphi \cos \psi) v+ \\
(\cos \varphi \sin \theta \sin \psi-\sin \varphi \cos \psi) w \\
\dot{z}=-(\sin \theta) \cdot u+(\sin \varphi \cdot \cos \theta) v+(\cos \varphi \cdot \cos \theta) w \\
\dot{u}=v \cdot r-w \cdot q-g \cdot \sin \theta+X_{u} \cdot u+(T / m) \cdot a \\
\dot{v}=w \cdot p-u \cdot r+g \cdot \cos \theta \cdot \sin \varphi+Y_{v} \cdot v+(T / m) \cdot b \\
\dot{w}=u \cdot q-v \cdot p+g \cdot \cos \theta \cdot \cos \varphi+T / m \\
\dot{\psi}=p+(\sin \varphi \cdot \tan \theta) \cdot q+(\cos \varphi \cdot \tan \theta) \cdot r \\
\dot{\theta}=(\cos \varphi) \cdot q-(\sin \varphi) \cdot r \\
\dot{\varphi}=(\sin \varphi / \cos \theta) \cdot q+(\cos \varphi / \cos \theta) \cdot r \\
\dot{p}=L_{a} \cdot a+L_{b} \cdot b \\
\dot{q}=M_{a} \cdot a+M_{b} \cdot b \\
\dot{r}=N_{r} \cdot r+N_{c o l} \cdot \delta_{\text {col }}+N_{p e d} \cdot \delta_{\text {ped }} \\
\dot{a}=-q-a / \tau+\left(A_{\text {lat }} / \tau\right) \cdot \delta_{\text {lat }}+\left(A_{\text {lon }} / \tau\right) \cdot \delta_{\text {lon }} \\
\dot{b}=-p-b / \tau+\left(B_{\text {lat }} / \tau\right) \cdot \delta_{\text {lat }}+\left(B_{\text {lon }} / \tau\right) \cdot \delta_{\text {lon }} \\
T / m=Z_{w} \cdot w+Z_{c o l} \cdot \delta_{\text {col }}-g
\end{gathered}
$$

where $x, y, z$ are position along ground axis; $u, v, w$ are velocities along body axis; $\psi, \theta, \varphi$ are Euler angles; $p, q, r$ are angular rates; $a, b$ are flapping angles. The inputs are lateral cyclic $\left(\delta_{\text {lat }}\right)$, longitudinal cyclic $\left(\delta_{\text {lon }}\right)$, tail rotor $\left(\delta_{\text {ped }}\right)$, and main rotor $\left(\delta_{c o l}\right)$. Coefficients for this model are shown in Table I. This identified model has successfully served the development of a model predictive controller for helicopter autonomous flight (Liu, 2010b).

\section{Table I here}

\subsection{Experiment setup}

\section{Figure 7 here}

Due to the limited payload of Trex-250 helicopter the use of onboard controller hardware was impractical. Instead, the helicopter was controlled by a desktop PC connected to a standard radio transmitter. To provide feedback for the controller, a Vicon Motion Capture system was used. This system includes eight cameras (Figure.7) that can 
cover a test volume of $5 \mathrm{~m} \times 4.5 \mathrm{~m} \times 2 \mathrm{~m}$ allowing enough room for Trex-250 maneuvering. With the knowledge of the relative positions of each camera and reflective ball attached to helicopter (Figure.6), Vicon system can determine the position and orientation of the helicopter. Furthermore, the parameter values of $p, q, r$ are estimated by Simulink block with orientation information.

\subsection{Control system design}

This paper presented here is an extension of work in $\mathrm{Fu}$ et al. (2011a) with an emphasis on chattering behaviour and approaching quality. Hence, the same cascade control structure in the previous paper is utilized here. The different point is that chattering-free UAS-SMC controllers are used here to replace the SMC controllers in $\mathrm{Fu}$ et al. (2011a). Here is the detail:

The control structure proposed in this paper is mainly based on the nested three loops architecture (Figure 8) in Dale et al. (2006). But some modifications are made because of the practical conditions. The flapping angles $a, b$ are immeasurable for Vicon system. Since the system in Equation (1) is a special system with the number of control input equal with that of states, the subsystems which have the similar property are expected in the architecture. Therefore, the attitude loop (Figure 9) is divided into three different loops which are Euler angle loop (Equation 17); angular rate loop (Equation 18); and rotor dynamic loop (Equation 19). In general, the architecture is composed of five loops as show in Figure 8 and Figure 9.

\section{Figure 8 here \\ Figure 9 here}

\subsection{Position Loop}

Since the design process for a whole control system in Trex-250 is too complex to show in this paper, position loop is used as an example to explain the design process with chattering-free UAS-SMC. The position loop is shown in Figure 10. Equations for this loop are given in Equation (15). In this subsystem, positions $x, y, z$ are given as states; velocities $u_{r e f}, v_{r e f}, w_{r e f}$ are given as control input. It means that the designed controller should send the reference velocities $u_{r e f}, v_{\text {ref }}, w_{\text {ref }}$ to next loop.

\section{Figure 10 here}

To avoid the complexity, formula $\dot{X}=f(X)+g(X) \cdot U$ is used to replace the Equation (15) where $X=[x, y, z]^{T}, U=\left[u_{r e f}, v_{r e f}, w_{r e f}\right]^{T}, f(X)=[0,0,0]^{T}$ 


$$
\begin{gathered}
g(X)=\left[\begin{array}{cc}
\cos \theta \cos \psi & \sin \varphi \sin \theta \cos \psi-\cos \varphi \sin \psi \\
\cos \theta \sin \psi & \sin \varphi \sin \theta \sin \psi+\cos \varphi \cos \psi \\
-\sin \theta & \sin \varphi \cos \theta \\
\cos \varphi \sin \theta \cos \psi+\sin \varphi \sin \psi \\
\cos \varphi \sin \theta \sin \psi-\sin \varphi \cos \psi \\
\cos \varphi \cos \theta
\end{array}\right]
\end{gathered}
$$

The chattering-free UAS-SMC equations for position loop in Trex-250 helicopter are given as follow:

From Equation (9), it is noted that the chattering-free UAS-SMC controller is expressed as

$$
U=g^{-1}(X)\left(-f(X)+\Omega_{1}^{-1} \cdot N-\Omega_{1}^{-1} \cdot \Omega_{2} \cdot X\right)
$$

where $\Omega_{1}=\operatorname{diag}\left\{\omega_{11}, \omega_{21}, \omega_{31}\right\}, \Omega_{2}=\operatorname{diag}\left\{\omega_{12}, \omega_{22}, \omega_{32}\right\}, N=\left[N_{1}, N_{2}, N_{3}\right]^{T}$

The item $N$ is the chattering-free robust function, $N_{1} \geq 0, N_{2} \geq 0, N_{3} \geq 0$

Coefficients $\omega_{21}, \omega_{22}, N_{2}, \omega_{31}, \omega_{32}, N_{3}$ used in $y, z$ channel are same with the coefficients $\omega_{11}, \omega_{12}, N_{1}$ in $x$ channel. Hence, only $x$ channel is discussed here. The switching surfaces for $X$ channel are expressed as

$$
S_{11}=0.7 x+\int x ; S_{21}=1.2 x+\int x
$$

\section{Table II here}

Current unidirectional auxiliary surface $H_{1}$ for $X$ channel is expressed as follow

$$
H_{1}=\omega_{11} x+\omega_{12} \int x+M_{1}
$$

where coefficients are shown in Table II. The chattering-free approaching law $N_{1}$ should be the key point to guarantee the continuity of control. The formula for $N_{1}$ is designed as follow:

$$
N_{1}= \begin{cases}N_{01}(x) & \text { when } x \text { in No. } 0_{i} \text { subsapce } \\ N_{11}(x) & \text { when } x \text { in No.1 } 1_{i} \text { subsapce } \\ N_{21}(x) & \text { when } x \text { in No. } 2_{i} \text { subsapce } \\ N_{31}(x) & \text { when } x \text { in No. } 3_{i} \text { subsapce }\end{cases}
$$


where $N_{01}(x)=\left\{\begin{array}{cc}\lambda_{01-} \cdot N_{01-}+\lambda_{-01-}\left(|x|+\left|\int x\right|\right) & x<0, \int x \geq 0 \\ 0.1 \cdot\left(|x|+\left|\int x\right|\right) & x<0, \int x<0 \\ \lambda_{01+} \cdot N_{01+}+\lambda_{-01+}\left(|x|+\left|\int x\right|\right) & x \geq 0, \int x<0\end{array}\right.$

$N_{11}(x)=\lambda_{11+} \cdot N_{11+}+\lambda_{11-} \cdot N_{11-}$

$N_{21}(x)=\lambda_{21+} \cdot N_{21+}+\lambda_{21-} \cdot N_{21-}$

$N_{31}(x)=\left\{\begin{array}{cc}\lambda_{31+} \cdot N_{31+}+\lambda_{-31+}\left(|x|+\left|\int x\right|\right) & x<0, \int x \geq 0 \\ 0.1 \cdot\left(|x|+\left|\int x\right|\right) & x \geq 0, \int x \geq 0 \\ \lambda_{31-} \cdot N_{31-}+\lambda_{-31-}\left(|x|+\left|\int x\right|\right) & x \geq 0, \int x<0\end{array}\right.$

$\lambda_{01-}=\left|\int x /(0.7 x)\right|, \lambda_{-01-}=1-\lambda_{01-}, \lambda_{01+}=\left|(1.2 x) / \int x\right|, \lambda_{-01+}=1-\lambda_{01+}$

$\lambda_{11+}=1-\left|\left(\left|\int x / x\right|-0.7\right) / 0.5\right|, \lambda_{11-}=1-\lambda_{11+}$

$\lambda_{21+}=1-\left|\left(\left|\int x / x\right|-0.7\right) / 0.5\right|, \lambda_{21-}=1-\lambda_{21+}$

$\lambda_{31+}=\left|(1.2 x) / \int x\right|, \lambda_{-31+}=1-\lambda_{31+}, \lambda_{31-}=\left|\int x /(0.7 x)\right|, \lambda_{-31-}=1-\lambda_{31-}$

$N_{01+}=0.2 x, N_{11_{-}}=0.152 x, N_{11+}=0.328 x, N_{31-}=0.2 x$

$N_{21-}=-0.152 x, N_{31+}=-0.2 x, N_{01-}=-0.2 x, N_{21+}=-0.328 x$

The gradients of switching surfaces $S_{11}, S_{21}$ is used to design the transformed weights

$$
\lambda_{01-}, \lambda_{-01-}, \lambda_{01+}, \lambda_{-01+}, \lambda_{11+}, \lambda_{11_{-}}, \lambda_{21+}, \lambda_{21_{-}}, \lambda_{31+}, \lambda_{-31+}, \lambda_{31-}, \lambda_{-31-}
$$

These weights are used to guarantee the continuity of function $N_{01}(x), N_{11}(x), N_{21}(x), N_{31}(x)$ and robust value $N_{01+}, N_{11-}, N_{11+}, N_{31-} N_{21-}$, $N_{31+}, N_{01-}, N_{21+}$ on the switching surfaces. Notice that the previous approaching law $N_{1}$ is not a unique form for chattering -free sliding mode. Every function $N_{1} \geq 0$ satisfied Equation (14) can be used to design a chattering-free control.

The chattering-free approaching law and coefficients for channel $y$ and $z$ can be designed in the same way. The design process for other loops except the rotor dynamic loop in Figure 8 would be same as in position loop.

\section{Analysis And Experiment Results}

\section{Figure 11 here}

The performance of Trex-250 while tracking the reference yaw angle from $0 \mathrm{rad}$ to $2 \pi \mathrm{rad}$ is shown in Figure 11. A spinning activity would be done by the Trex-250 helicopter 
under this reference signal. During this spinning activity, the helicopter was suffering the internal and external disturbance. Hence, it should be a good way to test the approaching quality of UAS-SMC and SMC strategies.

The position performance of Trex-250 is shown in Figure 12 14. The European coordinate system is used in this paper. Therefore, it is positive downwards for Z direction. There are two vertical lines annotated in Figure 12 and Figure 13. The left vertical line indicates that the helicopter is taking off at 20 seconds. And the right vertical line corresponds to the time when helicopter starts to spin.

\section{Figure 12 here}

From Figure 12, it is noted that helicopter is suffering an error up to $-0.2 \mathrm{~m} \sim+0.25 \mathrm{~m}$ with conventional SMC control while spinning. This is because that the approaching law $N_{i}, i=1, \cdots, n$ are zero on the switching surfaces $S_{i}$ for conventional SMC as shown in Figure 2. Hence, the helicopter shows a side -to-side oscillation. The error of UAS-SMC appears much smaller than the error of SMC. There also does not exist an obvious oscillation for UAS-SMC as shown in Figure 12. This is because the approaching law is not zero on the switching surface for chattering-free UAS-SMC as shown in Figure 5. Hence, the UAS-SMC demonstrates a better approaching quality than the conventional SMC method.

A drifting phenomenon can be found for the UAS-SMC strategy when the helicopter starts to take off. This is a normal phenomenon caused by the ground effect and the sit angle of Trex-250. Actually, it can be alleviated by a well-tuned UAS-SMC controller.

\section{Figure 13 here \\ Figure 14 here}

A similar performance for y direction of SMC and UAS-SMC is shown in Figure 12. The UAS-SMC controller shows better approaching quality than the SMC controller.

Since the spinning activity is happened in the $x, y$ direction, the $Z$ direction of Trex250 surfers less disturbance. The performance of UAS-SMC method in Figure 14 is similar with the performance of SMC strategy. The final reference signal for $Z$ direction is $0.2 \mathrm{~m}$ as given in Figure 14. Based on the past experiments, helicopter would hover at about $-0.1 \mathrm{~m}$ when the reference signal is $0 \mathrm{~m}$. Because of the ground effect, the lift of helicopter is higher than what we expected. Hence, helicopter should be forced to land down by $0.2 \mathrm{~m}$ reference signal.

\section{Conclusion}

The performance for a newly proposed chattering-free UAS-SMC controller is evaluated and experimentally tested on a TRex 250 helicopter in this paper. After introducing this chattering free sliding model control scheme, its robustness and approaching property has been analysed. This new control scheme is implemented on a small scale autonomous helicopter. A cascade structure is adopted to divide the flight control design for the helicopter into five interconnected loops, and for each loop, the corresponding controller is designed using the UAS-SMC method. For the purpose of the comparison, the 
conventional SMC is also implemented on the helicopter. Experimental results show that a much improved performance has been achieved by the UAS-SMC.

\section{Acknowledgment}

This work was carried out using the Vicon-Nexus tracking systems at Loughborough University.

Thanks to Jonathan H.A. Clarke, and Cunjia Liu for their technical support during the experiment.

\section{References}

A. Gaaloul and F. M’ Sahli. (2009). High gain observer-based higher order sliding mode controller. Proceedings of the 7th Asian Control Conference: 1713-1718.

Allamehzadeh, H. and Cheung, J.Y. (2003). Smooth response sliding mode fuzzy control with intrinsic boudary layer. Fuzzy Systems, 2003. FUZZ'03. The 12th IEEE International Conference: 488-493.

Bartibkubu, G. and Ferrara, A., Utkin V.I. (1995). Adaptive sliding mode control in discrete-time systems.Automatica, 31(5): 769-773.

Chen, M. S. and Yean-Ren, H., Masayoshi, T. (2002). A State-Dependent Boundary Layer Design for Sliding Mode Control. IEEE Trans., 47(10): 1677-1681.

Dale Enns, Tamas Keviczky (2006). Dynamic Inversion Based Flight Control for Autonomous RMAX Helicopter. Proceedings of the 2006 American Control Conference Minneapolis, Minnesota, USA.

Francesco, D. and Antonella, F. (2009). Higher Order Sliding Mode Controllers With Optimal Reaching. IEEE Trans., 54(9): 2126-2136.

Fu, J. and Chen, W. H., Wu, Q. X. (2011). Sliding mode control for a miniature helicopter. Proceedings of the 17th International Conference on Automation \& Computing:98-103. Fu, J. and Wu, Q., Jiang, C.(2011). Robust sliding mode control with unidirectional auxiliary surfaces for nonlinear system with state constraints. Control and Decision, 26(9): 1288-1294.

Fu, J. and Wu, Q. X., Jiang, C. S. (2011). Robust sliding mode positive invariant set for nonlinear continuous system. ACTA AUTOMATICA SINICA, 37(11) :1395-1401.

J,J. Slotine and S.S. Sastry (1983). Tracking control of non-linear system using sliding surfaces with application to robot manipulators. International Journal of Control, 38(29): 465-492

Lei, M. and Guo, W. A, Zhang, Y. W. (2010). Design of Time-Variable Boundary Layer on Sliding Mode Control. Proceeding of the 8th World Congress on Intelligent Control and Automation: 1539-1542.

Liu, C. J. and J. Clarke, Chen W. H. (2010). Modeling and identification of a minature helicopter. Proceeding of Workshop on Human Adaptive Mechatronics (HAM).

Liu, C. J. and Chen W. H., J. Andrews (2010). Model predictive control for autonomous helicopters with computational delay in consideration. in UKACC 2010.

Michael, D. and Thieery, F., Annemarie, K., Wilfrid, P. (2008). A novel higher order sliding mode control scheme. Systems \& Control Letters, 58(2): 102-108.

Wei, W. and Kenzo N., Yuta O. (2008). Model Reference Sliding Mode Control of Small Helicopter X.R.B based on Vision. International Journal of Advanced Robotic Systems, 5(3): 235 242.

Yang, L. and Fan, L., Lv, J. (2008). The quasi sliding mode control with varying boundary layers for a trolley inverted pendulum system. Control and Decision Conference, 2008. CCDC 2008: 4497-5000. 
Yigeng Huangfu, S. Laghrouche and Weiguo Liu (2010). A Chattering Avoidance Sliding mode Control for PMSM Drive , $20108^{\text {th }}$ IEEE International Conference on Control and Automation: 2082-2085.

\section{About the authors}

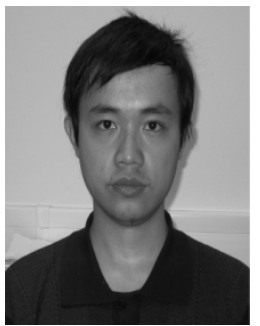

FU Jian, .........Email: fujian1986216@126.com

Ph. D. candidate at the College of Automation, Nanjing University of Aeronautics and Astronautics. He received his bachelor degree from Nanjing University of Science and Technology in 2007. His research interest covers sliding mode variable structure control and flight control. Fu is the corresponding author and can be contact.

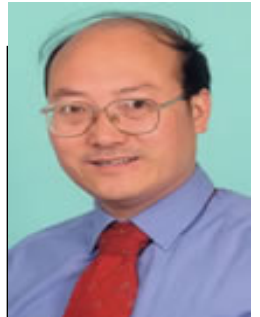

Chen Wen-Hua, ......

Wen-Hua Chen received his M.Sc. and Ph.D. degrees from the Department of Automatic Control at Northeastern University, China, in 1989 and 1991, respectively. From 1991 to 1996, he was a Lecturer in the Department of Automatic Control at Nanjing University of Aeronautics and Astronautics, China. He held a research position and then a Lectureship in control engineering in the Centre for Systems and Control at University of Glasgow, UK, from 1997 to 2000. He currently holds a Senior Lectureship in flight control systems in the Department of Aeronautical and Automotive Engineering at Loughborough University, UK. He has published three books and more than 150 articles in journals and conferences. His research interests include the development of advanced control strategies and their applications in aerospace engineering, particularly in unmanned aircraft systems. 


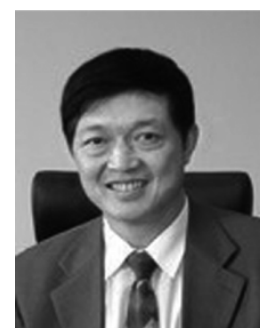

WU Qing-Xian, ......

Professor at Nanjing University of Aeronautics and Astronautics. He received his master degree from Northeastern University (China) in 1991.

His research interest covers industrial automatic, robust control and intelligent control. 
Table I. Coefficients for Mathematical helicopter.

\begin{tabular}{|ll|ll|}
\hline Parameter & Identified value & Parameter & Identified value \\
\hline $\mathrm{X}_{\mathrm{u}}$ & -0.233 & $\mathrm{Y}_{\mathrm{v}}$ & -0.329 \\
\hline $\mathrm{Z}_{\mathrm{w}}$ & -0.878 & & \\
\hline $\mathrm{L}_{\mathrm{a}}$ & $\mathrm{L}_{\mathrm{b}}$ & 745.67 \\
\hline $\mathrm{M}_{\mathrm{a}}$ & $\mathrm{M}_{\mathrm{b}}$ & 11.03 \\
\hline $\mathrm{T}$ & 53.98 & $\mathrm{~N}_{\mathrm{r}}$ & -23.98 \\
\hline $\mathrm{A}_{\text {lat }}$ & $\mathrm{A}_{\text {lon }}$ & 1.945 \\
\hline $\mathrm{B}_{\text {lat }}$ & 0.045 & $\mathrm{~B}_{\text {lon }}$ & -0.38 \\
\hline $\mathrm{Z}_{\text {col }}$ & 0.196 & & \\
\hline $\mathrm{N}_{\text {col }}$ & 2.120 & $\mathrm{~N}_{\text {ped }}$ & 113.65 \\
\hline
\end{tabular}

Table II. Coefficients for the x channel in position loop.

\begin{tabular}{|c|c|c|c|}
\hline$\omega_{11}$ & $\omega_{12}$ & $\mathrm{M}_{1}$ & Switching surfaces \\
\hline 1 & 1 & 1 & $S_{x 1}<0, S_{x 2}<0$ \\
\hline-0.44 & -0.2 & 1 & $S_{x 1}<0, S_{x 2} \geq 0$ \\
\hline 0.44 & 0.2 & 1 & $S_{x 1} \geq 0, S_{x 2}<0$ \\
\hline-1 & -1 & 1 & $S_{x 1} \geq 0, S_{x 2} \geq 0$ \\
\hline
\end{tabular}




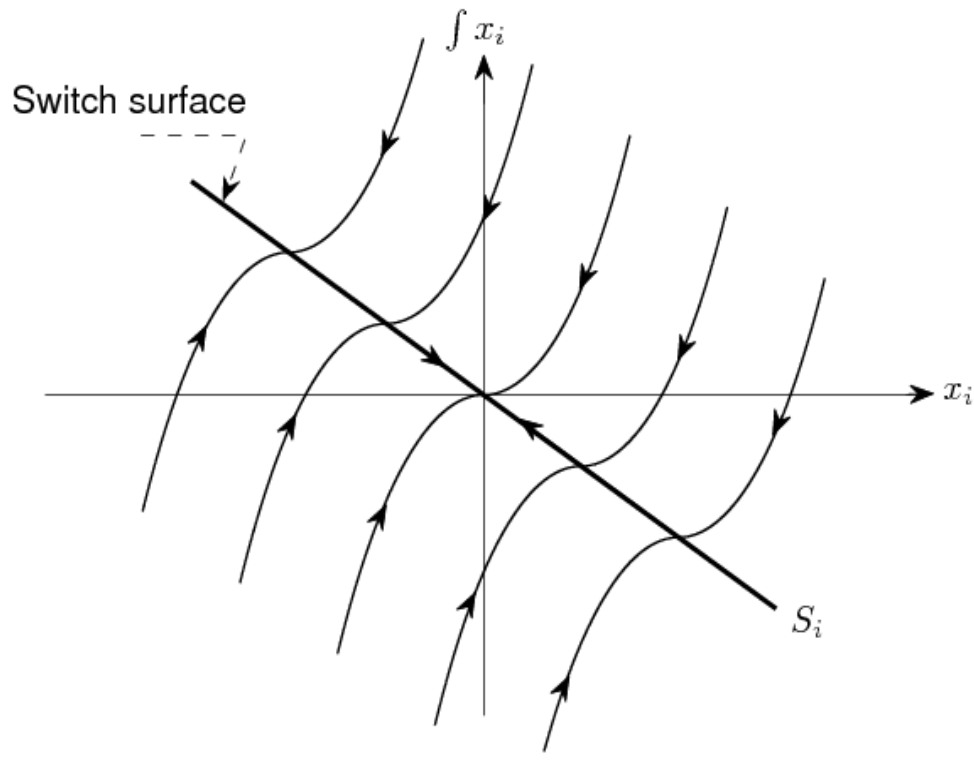

Figure. 1. Conventional sliding mode 


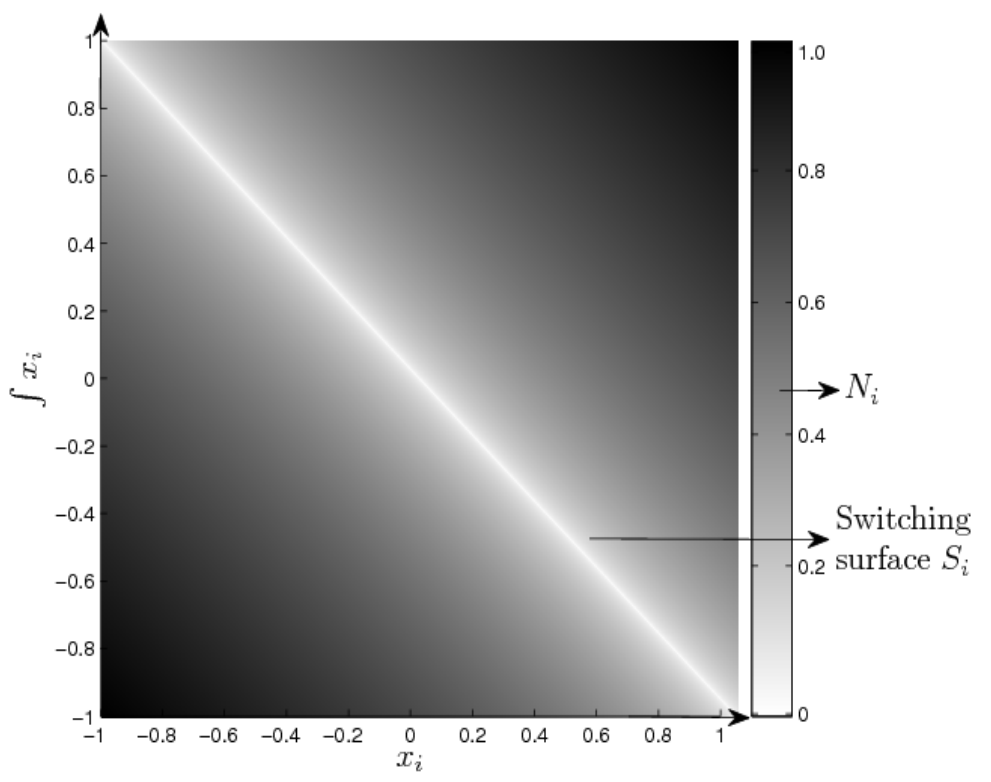

Figure. 2. Approaching quality $N_{i}$ in the conventional chattering-free sliding mode control 


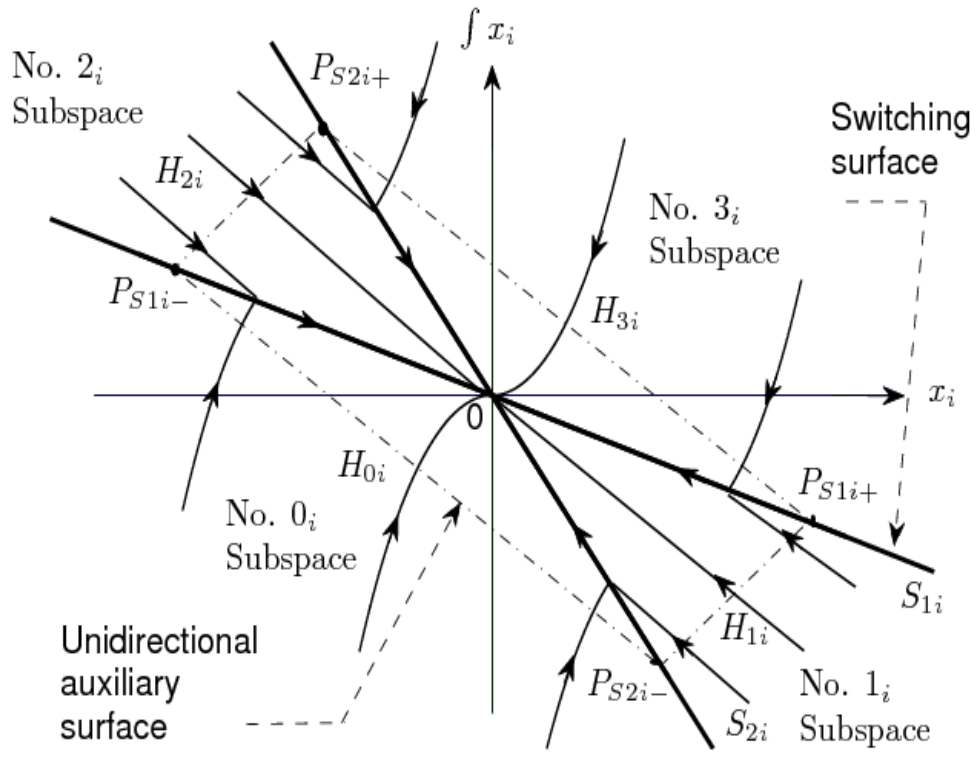

Figure. 3. UAS-SMC 


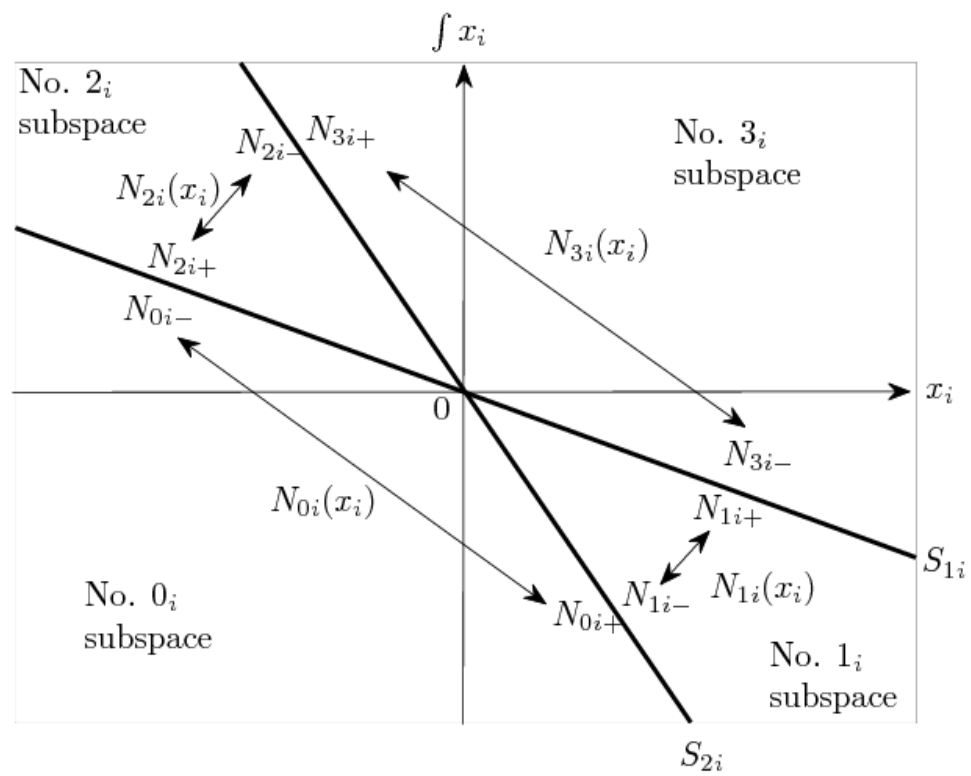

Figure. 4. Robust function in UAS-SMC 


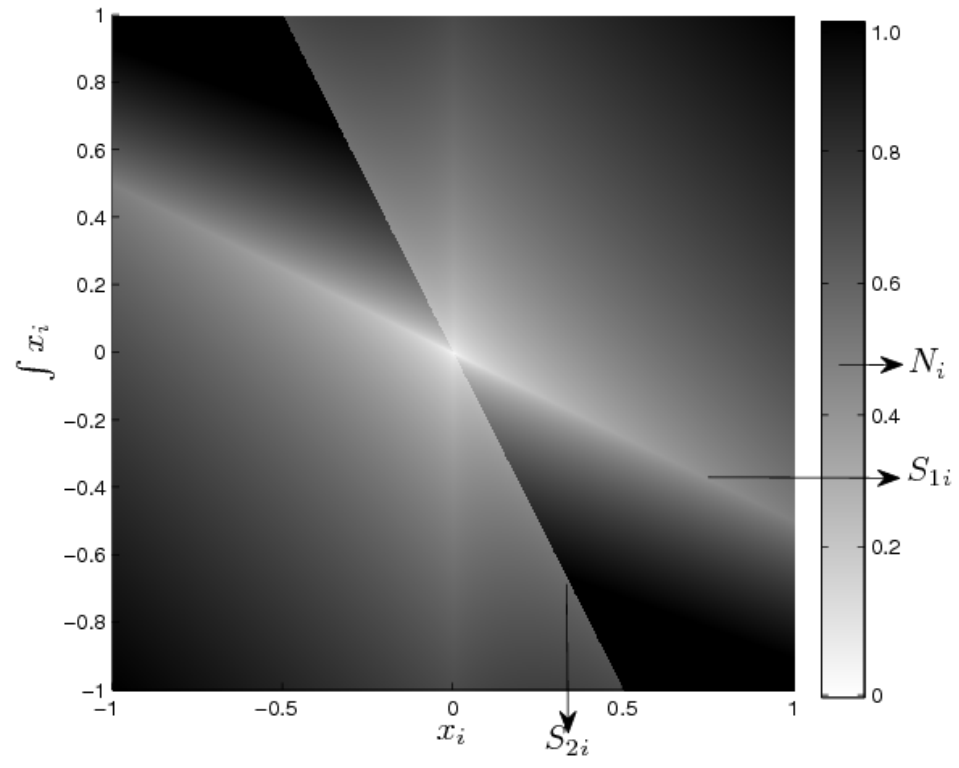

Figure. 5. Approaching quality $N_{i}$ in chattering-free UAS-SMC 


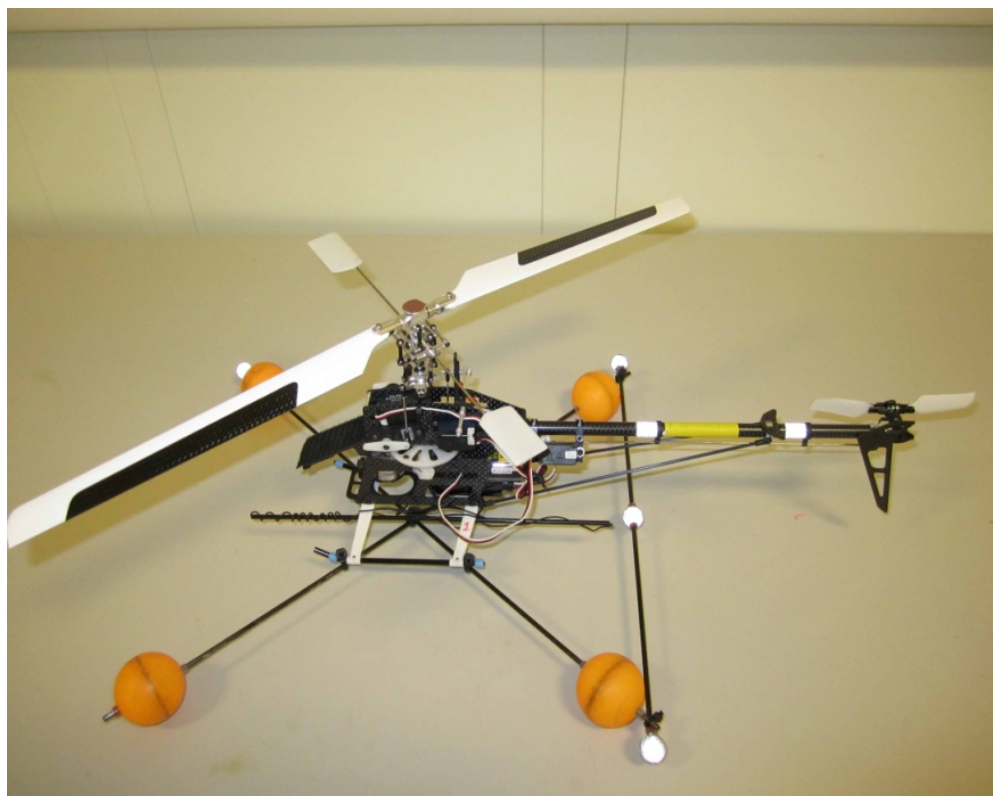

Figure. 6. Trex-250 Helicopter 


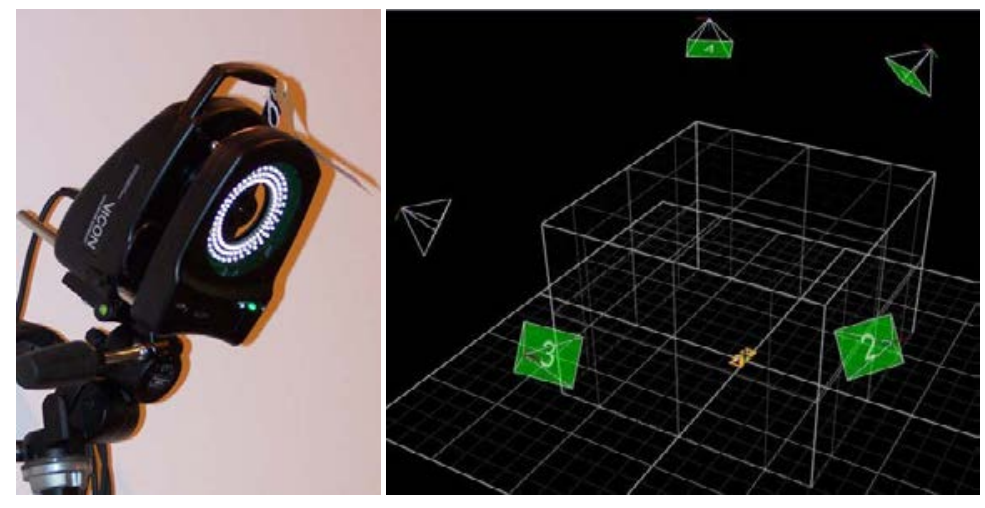

Figure. 7. Flight Test Environment 


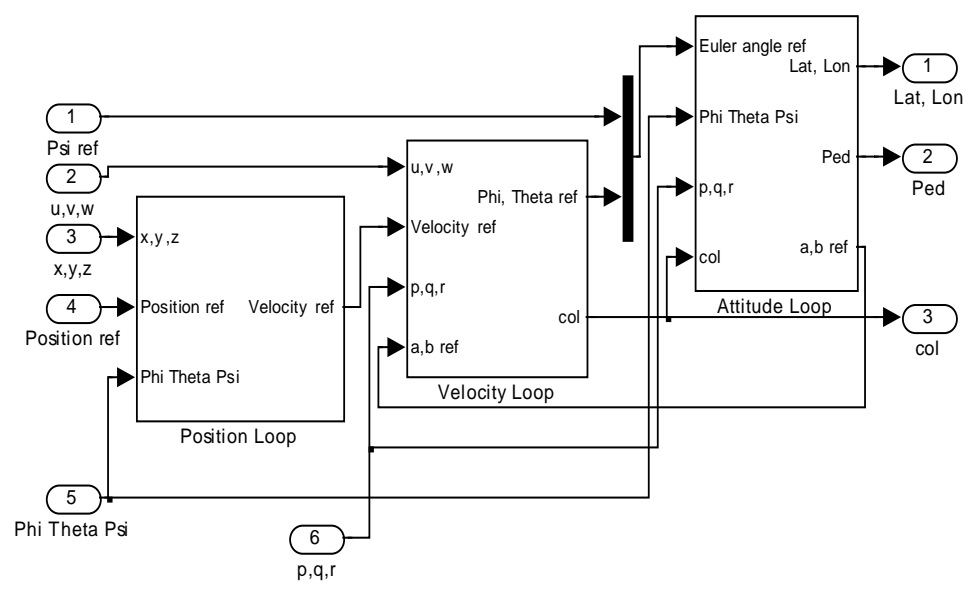

Figure. 8. Nested Loops 


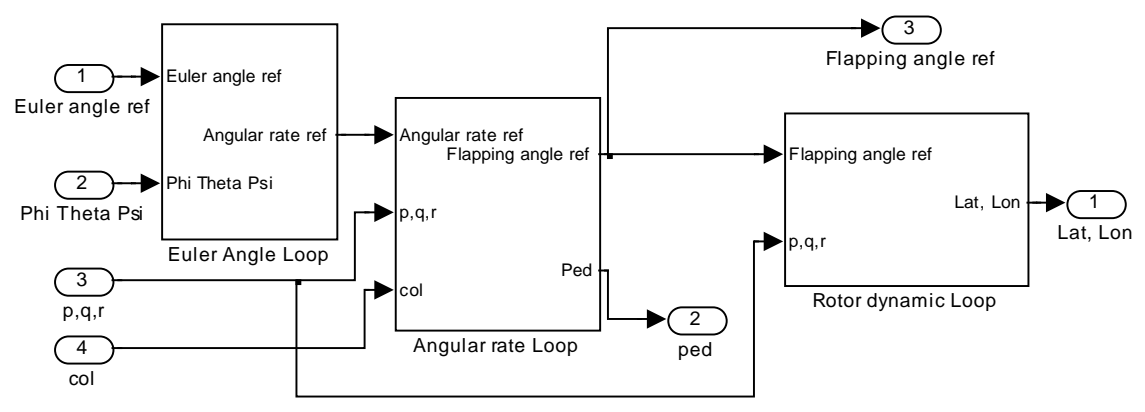

Figure. 9. Architecture of Attitude Loop 


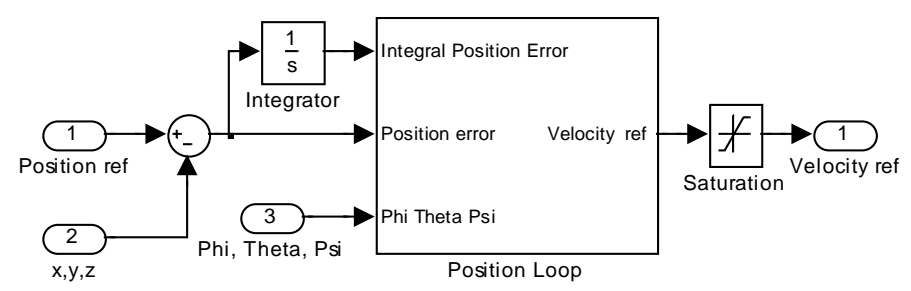

Figure. 10. Position Loop 


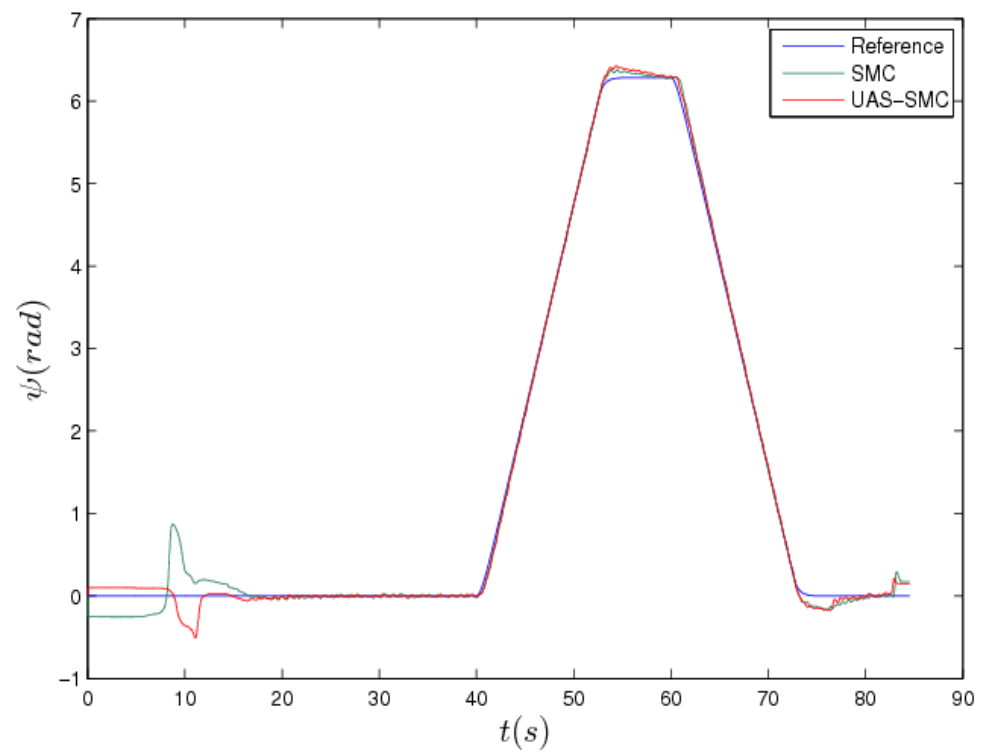

Figure. 11. Tracking the reference $\psi$ 


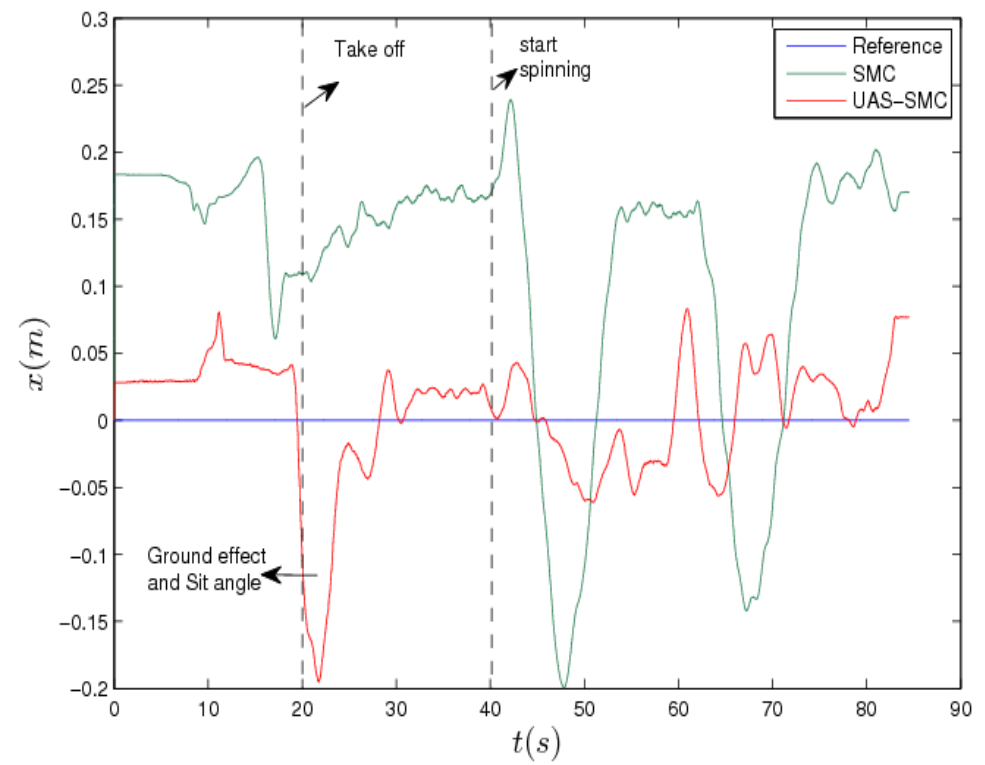

Figure. 12. $X$ Direction response of SMC and UAS-SMC 


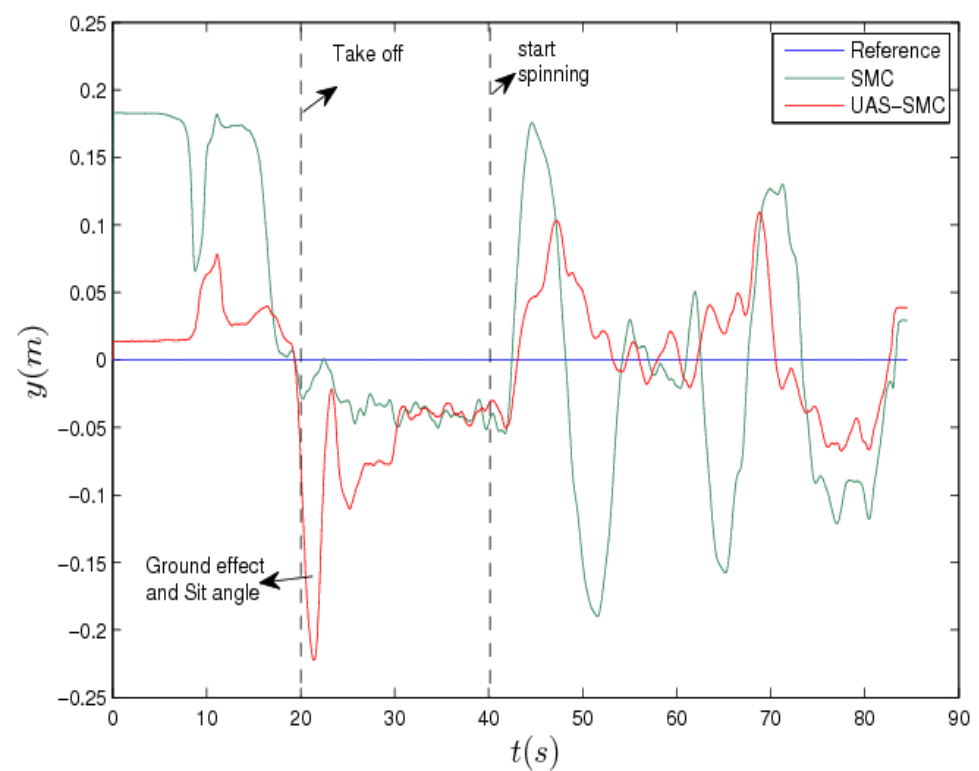

Figure. 13. $y$ Direction response of SMC and UAS-SMC 


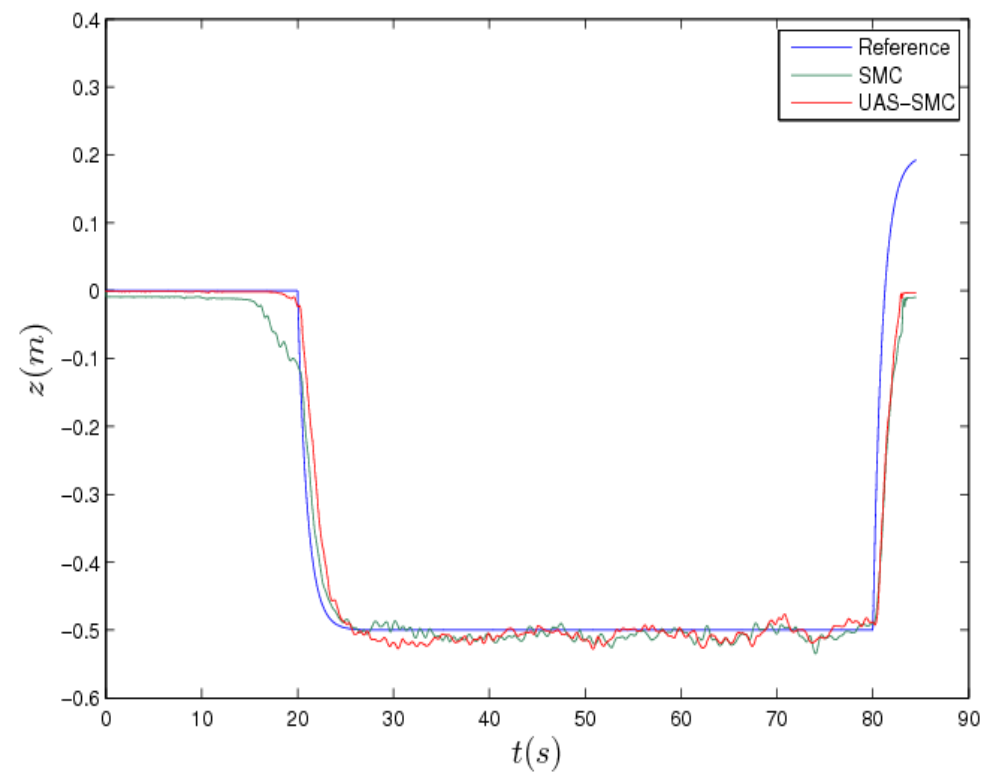

Figure. 14. $Z$ Z Direction response of SMC and UAS-SMC 